\title{
Oxygen, energy and light signalling direct meristem fate
}

Michael James Considine $e^{1,2,3,4,5}$

${ }^{1}$ School of Molecular Science, The University of Western Australia, Perth, 6009, Australia.

${ }^{2}$ School of Agriculture and Environment, The University of Western Australia, Perth, 6009, Australia.

${ }^{3}$ The UWA Institute of Agriculture, The University of Western Australia, Perth, 6009, Australia.

${ }^{4}$ Irrigated Agriculture Division, The Department of Primary Industries and Regional Development, South Perth, 6151, Australia.

${ }^{5}$ The Centre for Plant Science, University of Leeds, Leeds, LS2 9JT, United Kingdom.

Email. michael.considine@uwa.edu.au

Twitter. @vinebiology

Website. www.vinebiology.com

Key words. meristem, cell cycle, quiescence, stem cell, photomorphogenesis, TOR kinase, auxin, Nend rule, redox. 


\section{Abstract}

2 Light, nutrition and oxygen are each important cues for developmental transitions in plants. A small 3 number of recent studies have converged to give the first indication of how these environmental 4 cues act together and independently via auxin, cytokinin and ethylene to regulate cell proliferation 5 in the root and shoot meristems. 
7 Plants perceive and respond to environmental and nutritional cues to regulate meristem activity and

8 thus plant form. A number of developmental transitions also require certain abiotic conditions, and

9 one of the more well-studied examples is the light requirement for the skoto-photomorphogenic

10 transition in seedlings. Yet light incidence serves two purposes to plants, as signal and energy source, both of which may contribute to morphogenic responses. In turn, the energy from light via photosynthesis generates sugars and oxygen, both now known to function as signals for plant development, in addition to their metabolic roles (note; in the case of oxygen, it is low oxygen (hypoxia) that triggers signal transduction). A small number of important advances in the past 2-3 years have helped to disambiguate these pathways and develop an outline of how they interact to regulate plant development. As summarised here, the studies demonstrate that light- and sugardependent signals orchestrate meristem activity by converging on the TARGET OF RAPAMYCIN (TOR) kinase, which is a key sensory hub of energy status, and plays a direct role in driving cell proliferation. Moreover, the key components identified also implicate oxygen-dependent signalling, thus enabling new hypotheses of how cellular oxygen status influences proliferation by regulating TOR kinase (Figure 1).

Light is perceived by photoreceptors and pigments. Photoreceptors transduce light signals via hormone and transcription factor networks to enable photomorphogenesis, for example the apical hook response of the hypocotyl and subsequent development of true leaves. Pigments harvest photon energy to the photosystems for photosynthesis, which generates oxygen and sugars, themselves both fast-acting signals as well as primary substrates for downstream metabolism. A key regulator of the apical hook response is the E3 ubiquitin ligase CONSTITUTIVE PHOTOMORPHOGENESIS1 (COP1), which in the absence of light, targets photomorphogenic transcription factors for proteasomal degradation. Photoreceptor-mediated light signals negatively regulate COP1, enabling photomorphogenic transcription factors to accumulate and regulate the growth transition. As such, the Arabidopsis cop1 mutant displays constitutive photomorphogenesis even in darkness. Light-grown wild-type plants show a similar phenotype when photosynthesis is chemically inhibited. Although the cop1 mutant initiates true leaves in the dark, it is not capable of further organ proliferation. However, this phenotype is considerably rescued by supplementation with sucrose. Similarly, light alone is insufficient for the photomorphogenic transition in glucosestarved wild-type seedlings $[1,2]$. These insights show that both light and energy are necessary for photomorphogenesis.

Using the Arabidopsis cop1 background, Pfeiffer et al. [1] illustrated that the effects of light and sugars are transduced to the shoot apical meristem (SAM), regulating the expression of the stem cell identity gene WUSCHEL (WUS). The expression of WUS was additively dependent on sucrose and photoreceptor-mediated signalling but apparently did not require photosynthesis. The authors demonstrated that the sucrose-dependent expression of WUS was most likely an energy-related function, rather than sucrose-signalling per se, as glucose was also effective in inducing WUS, while the non-metabolisable palatinose was not. In contrast to the SAM, glucose was sufficient for activation of the cell cycle in the root apical meristem (RAM), where direct light was not necessary. These effects of glucose (in the SAM and RAM) and light (in the SAM) were dependent on the TOR kinase, which phosphorylates and activates the S-phase transcription factor E2Fa, enabling mitosis. Pfeiffer et al. [1] also demonstrated that the light signal was perceived distally from the SAM and relayed via cytokinin pathways. 
Remarkably, an independent study showed that the application of auxin was able to supplement the requirement for light to activate the SAM TOR kinase and enable the development of true leaves in wild-type Arabidopsis, when sucrose was present [2]. Hence, auxin acts downstream of photoreceptor-mediated light signalling to activate TOR kinase in the SAM, but sucrose or glucose are still required. Seedlings expressing a constitutively active form of a small GTPase Rho-related protein2 (ROP2) phenocopy the cop1, initiating the photomorphogenic transition in darkness. The authors demonstrated that the function of ROP2 required auxin, and it directly interacts with, and promotes TOR kinase activity. In contrast, glucose had no effect on ROP2. It was then shown that COP1 was indeed upstream of ROP2, and mediated by auxin [3]. The authors hypothesised that the distinction in light requirements for TOR kinase activity in the SAM and RAM was due to the auxin concentration in the respective apices; while continued auxin synthesis in the SAM requires light, the concentration in the RAM is constitutively sufficient [2].

\section{Oxygen and redox signalling may moderate light and energy signals}

The involvement of ROP2 in photomorphogenesis provides a logical link to oxygen and reduction/oxidation (redox) signalling [4]. The primary plant oxygen-signalling mechanism, the $\mathrm{O}_{2} /$ NO-dependent $\mathrm{N}$-end rule pathway of proteolysis ( $\mathrm{N}$-end rule) was recently described $[5,6]$. In conditions of hypoxia and low nitric oxide, Group VII ETHYLENE RESPONSE FACTORs (ERF-VII) are stable and transcriptionally activate genes involved in quiescence and etiolation pathways, which includes an increase in ethylene synthesis, starch hydrolysis, glycolysis and fermentation. In the presence of sufficient oxygen and nitric oxide, the N-end rule pathway targets the ERF-VIIs to the proteasome via the E3 ligase PROTEOLYSIS6 (PRT6). Fine regulation of ROP2 is required for survival and recovery of Arabidopsis following exposure to hypoxia, where redox homeostasis is disturbed [7]. ROP2 promotes synthesis of hydrogen peroxide $\left(\mathrm{H}_{2} \mathrm{O}_{2}\right)$ via NADPH oxidases (RBOH). Gonzali et al. [8] showed that a HYPOXIA RESPONSIVE UNIVERSAL STRESS PROTEIN1 (HRU1), which is transcriptionally induced in hypoxic conditions, directly interacts with ROP2, and likely also with $\mathrm{RBOH}$ and thioredoxin $\mathrm{h}$. HRU1 is consitutively elevated in plants where $\mathrm{N}$-end rule proteolysis is compromised, e.g. prt6 mutants or those expressing a constitutively stable ERF-VII, suggesting HRU1 is transcriptionally regulated by the ERF-VIls. Hence, the ERF-VII - HRU1 - ROP2 pathway provides a hypothetical means for oxygen and redox status to regulate TOR kinase and cell proliferation (Figure 1). Additionally, the increase in glucose resulting from of ERF-VII-dependent activation of starch hydrolysis provides an independent link between oxygen status and TOR kinase activity. Consequentially, hypoxic conditions should promote cell division.

Components of the $\mathrm{N}$-end rule pathway have also been shown to be essential for developmental transitions, including seed germination and photomorphogenesis [9]. Under hypoxic conditions, the apical hook of wild-type Arabidopsis remains closed, and similarly the apical hook of the prt6 mutant fails to fully open under normoxia [10]. Hence, an increase in oxygen availability as seedlings emerge through the soil is an important developmental signal. Interestingly, quiescence is a common consequence of hypoxia or conditions where ERV-VIIs are stable, e.g. seed dormancy or the SUB1A rice ([9] and references within). This raises the possibility that oxygen status can negatively regulate TOR kinase by a pathway yet to be defined (Figure 1).

The connection to ethylene signalling in photomorphogenesis also extends beyond the $\mathrm{N}$-end rule [11]. As indicated earlier, although cop1 mutants can initiate the photomorphogenic transition in the absence of light, they fail to proliferate and even penetrate the soil surface in certain conditions [12]. Overexpression of the central ethylene-dependent transcription factor ETHYLENE INSENSITIVE3 (EIN3), which is upstream of ERV-VIIs, partially rescues these deficiencies of cop1, because COP1 also 
mediates proteolytic destruction of EIN3 BINDING F BOX 1 and 2 (EBF1, 2), which in turn targets EIN3 for proteolysis.

\section{Concluding remarks}

Together these advances provide a significant integration of pathways previously thought to be largely independent in regulating plant development. As such, TOR kinase has emerged as a central hub for environmental and cellular cues which regulate the cell cycle, with several points of crosstalk between energy-, light- and oxygen-dependent pathways already established. Thus, these and related studies provide the required genetic tools and knowledge to explore a number of exciting questions. Firstly, does ERF-VII activity indeed promote TOR kinase independently via the ROP 2 and glucose pathways (Figure 1)? If so, how can the quiescence phenotypes such as seed dormancy and SUB1A rice be explained; do negative regulatory links, yet to be defined, exist between ERF-VII target genes and the TOR kinase (Figure 1)?

\section{Acknowledgements}

I would like to thank Dr Daniel Gibbs (Birmingham, UK), Prof Christine Foyer (Leeds, UK) and Prof John Considine (UWA, Australia) for critical comment, and Dr Santiago Signorelli (UWA, Australia) for formative discussions. This work was funded by the Australian Research Council (DP150103211) and Department of Primary Industries and Regional Development.

\section{References}

1. Pfeiffer, A. et al. (2016) Integration of light and metabolic signals for stem cell activation at the shoot apical meristem. eLife 5, e17023.

2. Li, X. et al. (2017) Differential TOR activation and cell proliferation in Arabidopsis root and shoot apexes. Proc Natl Acad Sci USA 114, 2765-2770

3. Cai, W. et al. (2017) COP1 integrates light signals to ROP2 for cell cycle activation. Plant Signal Behav DOI: 10.1080/15592324.2017.1363946 (www.tandfonline.com/toc/kpsb20)

4. Considine, M.J. and Foyer, C.H. (2014) Redox regulation of plant development. Antioxid Redox Signal 21, 1305-1326.

5. Gibbs, D.J. et al. (2011) Homeostatic response to hypoxia is regulated by the $\mathrm{N}$-end rule pathway in plants. Nature 479, 415-418.

6. Licausi, F. et al. (2011) Oxygen sensing in plants is mediated by an $\mathrm{N}$-end rule pathway for protein destabilization. Nature 479, 419-422.

7. Baxter-Burrell, A. et al. (2002) RopGAP4-dependent Rop GTPase rheostat control of Arabidopsis oxygen deprivation tolerance. Science 296, 2026-2028.

8. Gonzali, S. et al. (2015) Universal stress protein HRU1 mediates ROS homeostasis under anoxia. Nature Plants 1, 15151.

9. Considine, M.J. et al. (2017) Learning to breathe: Developmental phase transitions in oxygen status. Trends Plant Sci 22, DOI: 10.1016/j.tplants.2016.11.013 (www.cell.com/trends/plantscience).

10. Abbas, M. et al. (2015) Oxygen sensing coordinates photomorphogenesis to facilitate seedling survival. Curr Biol 25, 1483-1488.

11. Zhu, Q. and Benková, E (2016). Seedlings' strategy to overcome a soil barrier. Trends Plant Sci 21, 809-811.

12. Shi, H. et al. (2016) Seedlings transduce the depth and mechanical pressure of covering soil using COP1 and ethylene to regulate EBF1/EBF2 for soil emergence. Curr Biol 26, 139-149. 


\section{Figure legend}

Figure 1. Light incidence on plants serves a dual function of signal and energy source, which converge via hormone-, oxygen- and sugar-mediated pathways to regulate the cell cycle via the conserved energy sensor TARGET OF RAPAMYCIN kinase (TOR). Photoreceptors negatively regulate CONSTITUTIVE PHOTOMORPHOGENESIS1 (COP1), itself a negative regulator of key photomorphogenic transcription factors such as HY5 (TFs), as well auxin and ethylene signalling pathways, including the EIN3 BINDING F BOX 1 and 2 (EBF1/2). In the presence of light, auxin and the EBF1/2 pathways are activated. Auxin signalling activates a small GTPase Rho-related protein2 (ROP2), which triggers synthesis of hydrogen peroxide $\left(\mathrm{H}_{2} \mathrm{O}_{2}\right)$ via NADPH oxidases (RBOH). $\mathrm{H}_{2} \mathrm{O}_{2}$ feeds back to promote auxin signalling. ROP2 directly binds to and promotes TOR kinase, which phosphorylates and activates the core G1/S-phase transcription factor family of E2F, leading to the expression of the stem cell identity protein WUSCHEL (WUS). However the auxin-mediated pathway is insufficient without two sympathetic pathways, via oxygen and sucrose, both products of photosynthesis. Oxygen status is signalled via N-end rule proteolysis of Group VII ETHYLENE RESPONSE FACTORs (ERF-VII), which are negatively regulated by PROTEOLYSIS6* (PRT6) under normal oxygen conditions, or activate quiescence or etiolation pathways, including ethylene synthesis and starch hydrolysis ( $\alpha$-amylase) under low oxygen status (hypoxia). The ERF-VIls negatively regulate photomorphogenesis, and promote expression of HYPOXIA RESPONSIVE UNIVERSAL STRESS PROTEIN1 (HRU1), which in turn promotes ROP2. The ERF-VII may also influence TOR kinase by other pathways, although this has not been demonstrated. The ERF-VIIs are also negatively regulated by light, via the photoreceptor-mediated pathway through COP1-dependent degradation of EBF1/2. Sucrose, via glucose activates TOR kinase and other cell cycle regulators. Hence the cellular cues, energy, oxygen, auxin and ethylene act together and independently to mediate the photomorphogenic transition. $\mathrm{G} 1$ and $\mathrm{S}$ denote the respective phases of the cell cycle. Arrow with dashed lines are indirect relationships. *The dotted line between oxygen and PRT6 indicates that PRT6 is not regulated by oxygen per se (ref $[5,6,9])$. 


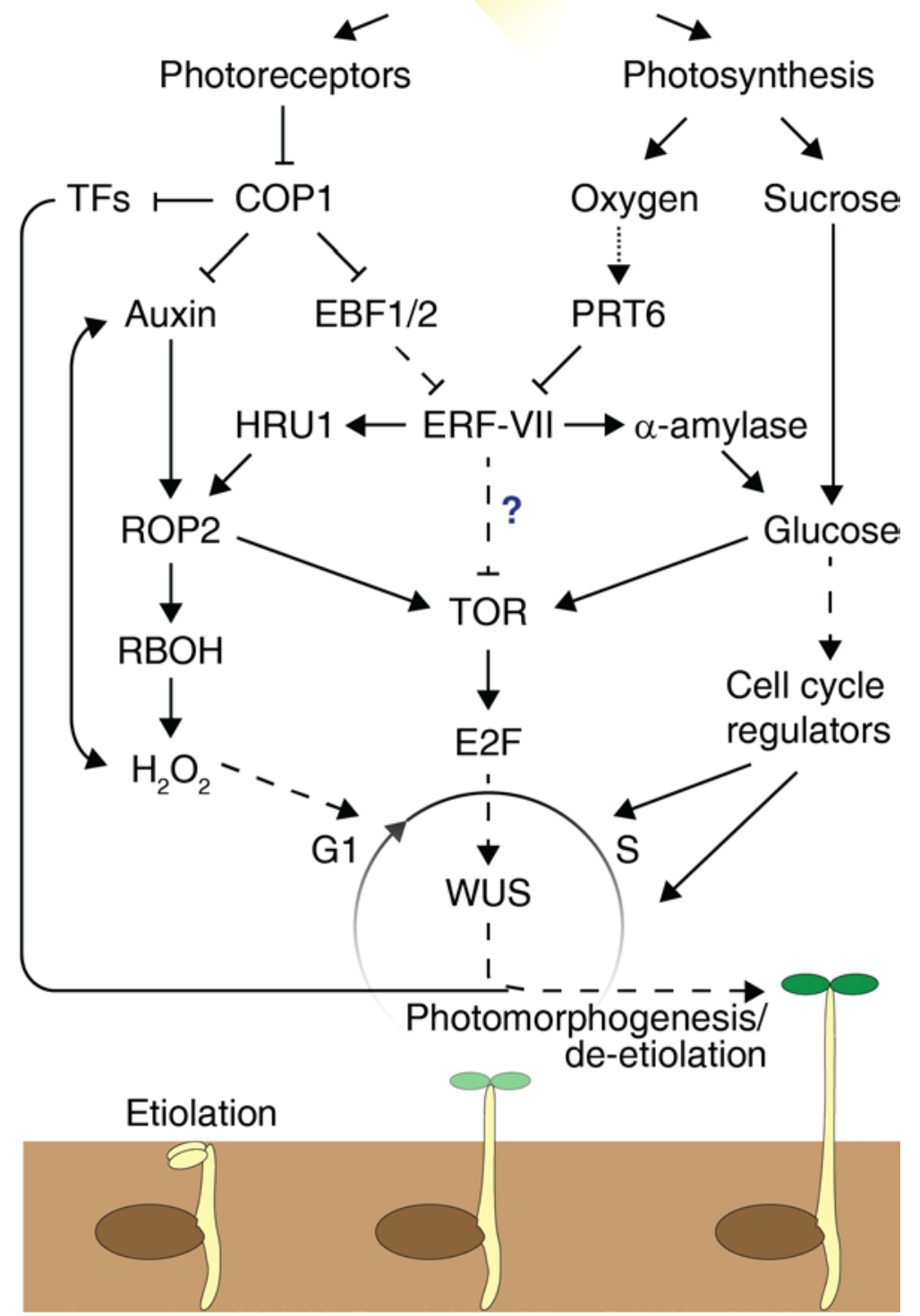

